\title{
The X-Ray Structure of the Ligand-Free Antibiotic Ristocetin-A Reveals the Role of the Monomer/Dimer Equilibrium in Its Binding Mode
}

\author{
Thierry Prangé and William B. T. Cruse \\ Laboratoire de Cristallographie et RMN Biologiques, Faculté de Pharmacie, UMR 8015 CNRS, \\ 4 avenue de l'Observatoire, 75006 Paris, France \\ Correspondence should be addressed to Thierry Prangé; thierry.prange@parisdescartes.fr
}

Received 25 March 2016; Accepted 17 May 2016

Academic Editor: Rajesh G. Gonnade

Copyright (C) 2016 T. Prangé and W. B. T. Cruse. This is an open access article distributed under the Creative Commons Attribution License, which permits unrestricted use, distribution, and reproduction in any medium, provided the original work is properly cited.

\begin{abstract}
Ristocetin-A belongs to the group of the glycoheptapeptide antibiotics. The sulfate salt of ristocetin-A was crystallized in the $\mathrm{P} 2_{1}$ monoclinic space group with a homodimer in the asymmetric unit. The two subunits are linked back-to-back like the other members of the family via four peptide bonds forming a twisted $\beta$-sheet and exposing the binding pockets to the exterior. The two tetrasaccharide parts of this ligand-free antibiotic are in the anti/anti orientations contrary to what was found in the monoand diliganded ristocetin-A/-(D-Ala-D-Ala) complexes in which the two tetrasaccharides of the dimer are syn/anti. The ligand-free dimer shows however some conformational differences between the two subunits, particularly in the terminal arabinose leading to one extended and one bent conformation of the tetrasaccharide moiety. Comparison between this structure and the two available mono- and diliganded structures confirms that the anti/anti to syn/anti flipping of the tetrasaccharide is a key step in the binding to the D-Ala-D-Ala-containing target sequence and cannot proceed without displacement of the monomer/dimer equilibrium.
\end{abstract}

\section{Introduction}

Ristocetin-A (Figure 1) is a highly oxidized glycopeptide antibiotic produced by an Amycolatopsis strain. It is a close parent of vancomycin. Being formerly considered as very promising against infections caused by Gram-positive bacteria, its use in antibiotherapy was discontinued because of risk of serious side effects on platelet aggregation. RistocetinA found however an important use in diagnosing anomalies of the plasma von Willebrand factor. The mechanism of action is mainly through interactions in the biosynthesis of bacteria cell wall. A number of D-Ala-D-Ala related peptides in complex with such glycopeptide antibiotics are available in the literature, not only for ristocetin-A [1] but also for vancomycin [2], balhimycin [3], or chloroorienticinA [4]. Some of them were reported in complex with a protein implicated in their biosynthesis like teicoplanin [5]. Recently, the association of a protein carrier allowed successful preparation of chimeric crystals-though not diffracting at atomic resolution - of new antibiotics like dalbavancin [6]. Currently, in the case of ristocetin-A, two X-ray structures are available. The first at atomic resolution is a biliganded structure with one independent monomer in a trigonal space group, in which the dimer is built around a twofold crystallographic axis, the second is a monoliganded structure, associated with a maltose binding protein at moderate resolution $(1.85 \AA$ ) [6]. In both cases, two ristocetin-A molecules (Figure 1) associate together to build a homodimer through main-chain peptide association at their back interface. In the case of the biliganded structure, the dimerization around a twofold crystallographic axis was likely to be responsible for the 50:50 syn/anti statistic disorder (i.e., the arabinose in opposite direction with respect to the $\mathrm{N}$-terminus of the peptide chain) observed for the tetrasaccharide. This structure holds two liganded D-Ala-D-Ala peptides in the two binding pockets, in a dissymmetric arrangement, the first with a high affinity, corresponding to the syn orientation of the saccharide, and the second with a lower affinity, which 


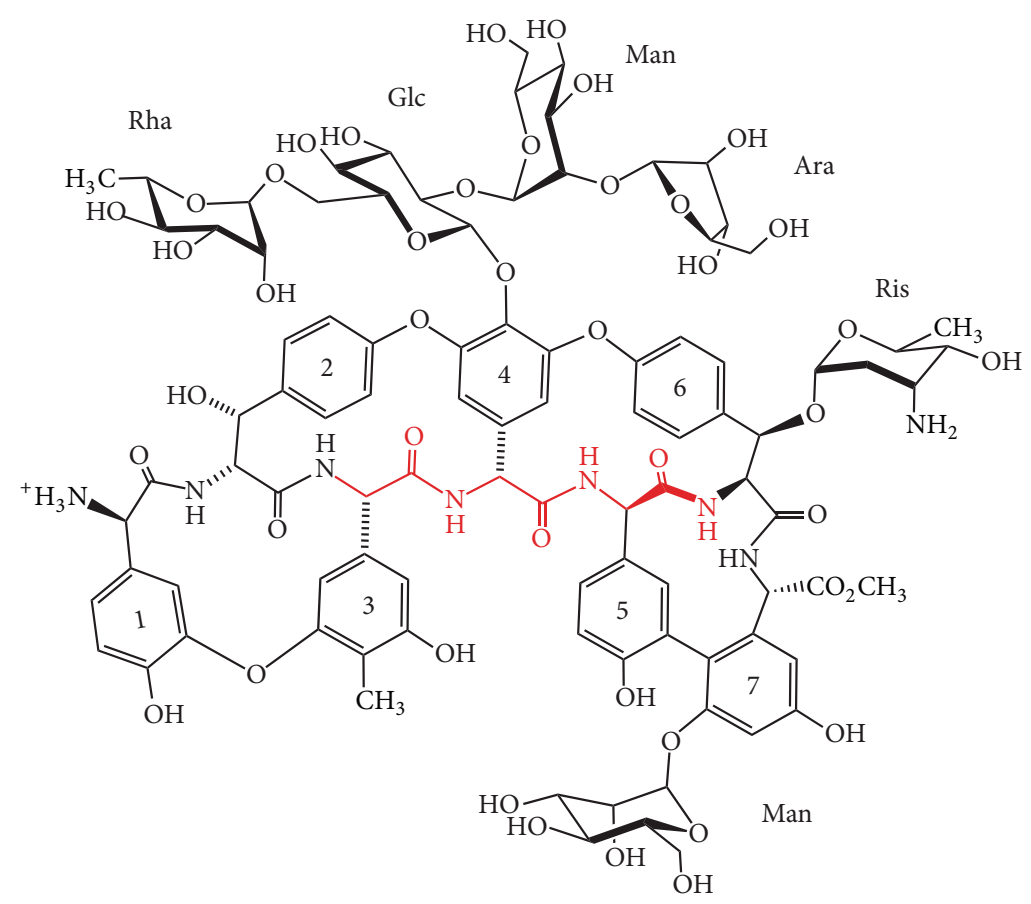

Figure 1: Chemical structure of ristocetin-A. The molecule dimerizes via four intermolecular $\mathrm{H}$ bonds (sequence in red). The asymmetric unit is a dimer: (ristocetin-A) ${ }_{2}{ }^{+}, \mathrm{SO}_{4}{ }^{2-}$. The amino-acid configurations are DDLDDLL from residues \#1 to \#7, respectively. The tetrasaccharide Rha-Glc-Man-Ara is represented in the anti orientation with respect to the glycosidic bond Glc-Tyr 4, that is, positioning the arabinose sugar near the glycosylated $\beta$-Tyr 6 residue.

corresponds to the anti orientation of the saccharide. In the second monoliganded structure, only the high affinity site of the dimer is occupied with the tetrasaccharide in the syn orientation. We present here the structure of the ligandfree sulfate salt of ristocetin-A, which is also a homodimer but without implication of any crystallographic axis and two perfectly defined tetrasaccharide motifs at atomic resolution, both in the anti orientation.

\section{Materials and Methods}

2.1. Crystallizations. It is well known that the ligand-free glycopeptides are difficult to crystallize as such without the presence of a ligand. As mentioned above in addition to standard crystallization techniques based on vapor diffusion in presence of a specifically designed ligand, a new approach was developed using a carrier protein containing at the Cterminus the target sequence Lys-(D-Ala $)_{2}[6,7]$. However, in the case of the ligand-free sulfate salt of ristocetin-A, we have been able to obtain large crystals simply by slow vapor diffusion of acetone in a water solution of the antibiotic sulfate salt. Ristocetin-A sulfate was purchased from Sigma-Aldrich as a white powder. A ristocetin-A solution $(100 \mathrm{mg} / \mathrm{mL}-3 \mathrm{~mL})$ was set in a small hemolysis glass tube $(11.6 \times 75 \mathrm{~mm})$ and the tube was placed vertically in a closed flask containing at the bottom a large quantity of acetone $(20 \mathrm{~mL})$. In about two days, acetone diffusion in the tube causes the volume of the solution in the tube to increase two to three times and leads to bulk crystallization of ristocetin-A along the tube wall. The first crystal crop usually gives crystals of poor quality due to the small amount of ristocetin-B acting as a contaminant. These crystals were filtered, redissolved, and set again to the same crystallization conditions. This second round leads to perfect crystals that were now used in diffraction recordings. Depending on how slowly acetone diffuses in the ristocetin-A solution, the crystals can reach in favourable circumstances one $\mathrm{mm}$ in size. Once formed, they were rapidly harvested in nylon loops for flash-cooling in liquid nitrogen. As the crystals contain a large amount of acetone, it was not necessary to use any cryoprotectant before the quenching step.

2.2. Data Collection and Processing. Data collections were recorded at the synchrotron LURE Laboratory, Orsay, France, on beam line W32. A single crystal was used for two complete $360^{\circ}$ rotations, one rapid at moderate resolution to get the strongest reflexions unsaturated and another slow rotation at the minimum distance to get accurate reflexions at the highest resolution. Due to geometry considerations of the experimental setup and the wavelength fixed at $0.964 \AA$, the resolution at the edge of the MAR-345 image-plate detector was limited to 1.0 A but diffraction could be noted beyond this limit. The two data sets were processed, scaled, and merged with the CCP4 package of programs [8]. The final set of unique reflexions was reformatted for compatibility with the SHELX suite of programs [9].

2.3. Structure Determination and Refinements. The data set was used as a successful test case during the development of the SHELXD program [10]. We started with the vancomycin 
TABLE 1: Data collection and refinements statistics (values for the highest resolution bin are in parentheses).

\begin{tabular}{lc}
\hline Data collection & \\
Wavelength $(\AA)$ & 0.964 \\
Space group & $\mathrm{P} 2_{1}$ \\
& $a=23.850(1) ;$ \\
Unit-cell parameters $\left(\AA{ }^{\circ}{ }^{\circ}\right)$ & $b=26.951(2) ;$ \\
& $c=26.389(1) ;$ \\
Resolution range $(\AA)$ & $\beta=117.17(4)$ \\
Number of measured reflections & $10-1.0(1.12-1.0)$ \\
Number of unique reflections & $40123(7221)$ \\
Completeness $(\%)$ & $15224(3642)$ \\
Mean $I / s(I)$ & $93.8(78.6)$ \\
$R_{\text {merge }}(\%)$ & $18.6(4.2)$ \\
Refinements & $4.3(19.5)$ \\
Resolution range $(\AA)$ & \\
Number of refined atoms & $10-1.0$ \\
Number of observed reflections $(F)$ used & 401 \\
Mean $B$ values $\left(\AA^{2}\right)$ & 15116 \\
$\quad$ Ristocetin-A-aglycon & \\
Ristocetin-A-saccharides & 4.2 \\
Acetone & 5.3 \\
Water & 10.7 \\
$R$ (obs. $F s)$ & 17.2 \\
w $R_{2}$ (obs. $F^{2}$ s) & 0.1147 \\
CCDC deposition number & 0.3034 \\
\hline
\end{tabular}

aglycon [11], or better with the aglycon of balhimycin [3], as a rigid body seed (GROP instruction in SHELXD). This gave a sharp and contrasted solution with all the atoms of the aglycon and part of the sugars. The full structure was completely developed in a single run with the automated DIRDIF recycling procedure based on E-maps [12]. Refinements were done with the SHELXL/H programs [9] with isotropic and then anisotropic thermal parameters for non- $\mathrm{H}$ atoms. Constraints on 1-2 and 1-3 distances as well as on planar rings were applied throughout but chiral volumes (zero and nonzero) were not constrained. During refinements, ten molecules of acetone were localized in addition to a number of water molecules with full or partial occupancies. In the last rounds of refinement, hydrogen atoms were introduced at their theoretical places with an isotropic thermal factor riding on that of the bonded atom. Final statistics are reported in Table 1. The structure is depicted in Figure 2(a) (the monomer) and Figure 2(b) (the complete homodimer of the a.u.). All figures were drawn using PyMol [13].

\section{Results}

3.1. The Aglycon. The aglycon part of ristocetin-A (Figure 2(a)), common to most of the glycopeptide antibiotics (vancomycin, eremomycin, balhimycin, etc.), is a rigid core of seven residues all deriving from highly oxidized Phe and Tyr residues, like nor- $\beta$-Tyr or $\beta$-hydroxylated Tyr with four of them in the $\mathrm{D}$ configuration, a classic way for microorganisms to resist peptidases from other competitors. These residues are assembled in a four-ring system forming a compact and curved template with a methylated C-terminus (Figure 1 ). The main peptide chain points to the convex face, the active site being located on the concave face of the aglycon. Two aglycon subunits associate together by their convex faces to form a homodimer around a local noncrystallographic twofold axis, through four $\beta$-sheet-like hydrogen bonds (Figure 2(b)). Within the dimer, the two aglycon conformations are identical because of the high rigidity of the four-ring system; the rms between the two is $0.06 \AA$, resulting from a nearly perfect twofold rotation. This is obviously the case in the diliganded form, which uses the crystallographic twofold axis of the trigonal $\mathrm{P} 3_{1} 21$ space group to generate the dimer. However, comparisons of the aglycons in the bound and unbound forms of ristocetin-A show they are slightly different, with a ligand-free aglycon more open than the occupied aglycon in liganded structures.

3.2. The Tetrasaccharide. The tetrasaccharide Rhap-GlcpManp-Araf linked to ring \#4 on the top of the monomer delimits one of the rims lining the binding cavity. Resulting from the convex face dimerization, the two sites on the concave faces are exposed to the solvent. The two copies of the tetrasaccharide located on top of the dimer face each other in a head-to-tail or anti/anti association (Figure 3(b)). This is in contrast with the mono- and diliganded forms of ristocetin-A where the two tetrasaccharides are syn/anti oriented. This leads to a steric hindrance at this level of sugar-sugar interactions and the difficulties resulting from the statistic distribution of the syn and anti orientations reported in reassigning the sugar groups in the diliganded structure [14] were not observed here. In the present anti/anti association, the sugars are all well defined in both subunits (Figure 2(c)) with the end-arabinose clearly in the furanose conformation. The two anti saccharides have however slightly different conformations when compared to each other (Figure 3(a)), mainly flipping of the arabinose sugar (arrow in Figure 3(a)). This leads to two anti conformations that can be described as "extended" (open) and "bent" (or closed), both corresponding to the conformation $a$ described in the biliganded structure [1] and to the orientation in the free subunit of the monoliganded dimer/MBP structure [6].

In the asymmetric unit, there is one homodimer for one sulfate ion, thus resulting in a monoprotonated ristocetin-A molecule, probably at the $\mathrm{N}$-terminal amino group preferably to the amino group of the ristosamine connected to residue \#6.

3.3. Ristocetin-A: Ligand-Free Structure versus Liganded Structures. It was postulated since a couple of decades that the syn/anti pivoting of the saccharide unit would play a crucial role in the bacterial wall binding [15]. In the case of vancomycin or other glycopeptide antibiotics with shorter sugar sequences, the pivoting of the saccharide would be easy to do within the dimer, but in the case of ristocetin-A, this step would be hard to perform without dissociating the dimer. 


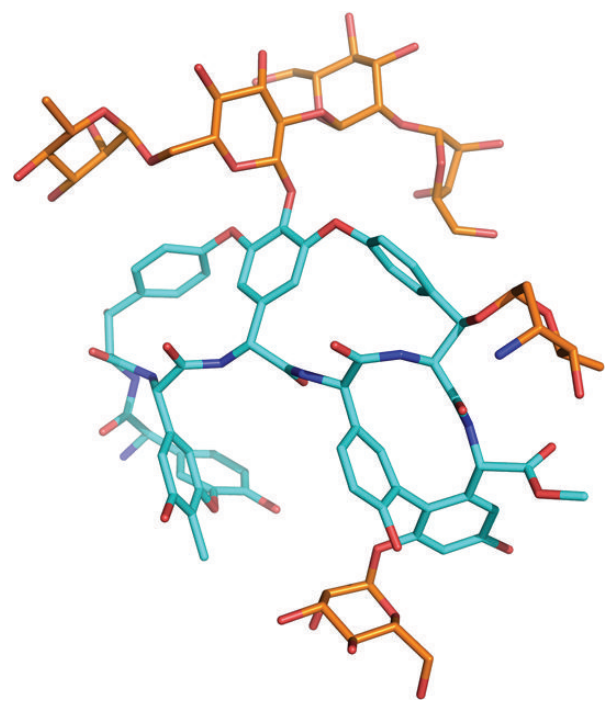

(a)

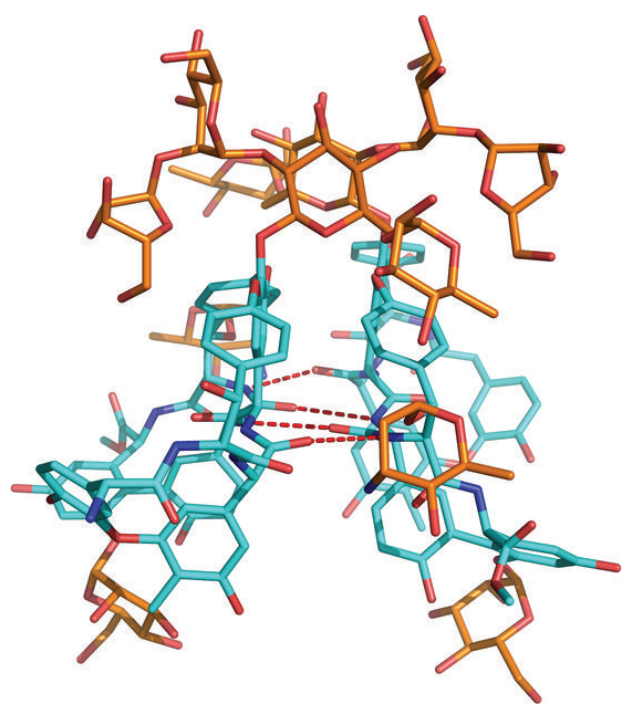

(b)

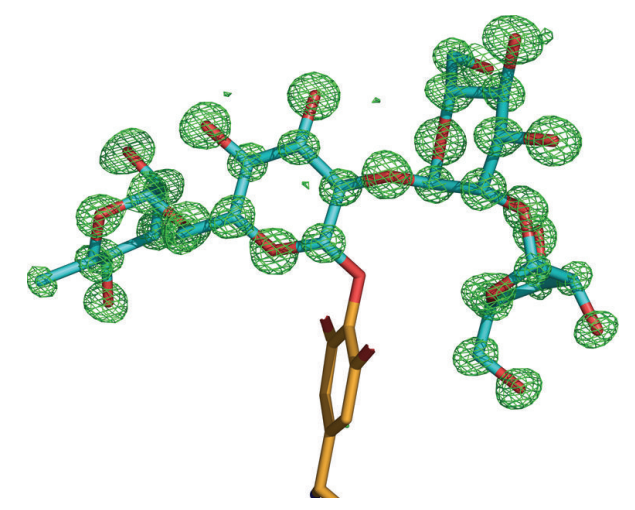

(c)

Figure 2: (a) The monomer of ristocetin-A. The aglycon is in blue and the sugars are in orange. (b) The dimer of the asymmetric unit. The local twofold axis building the dimer is vertical in the plane of the figure. The four hydrogen bonds of the short $\beta$-sheet are represented by red dotted lines. Acetone and water molecules are not reported for clarity. (c) Electron density (omit-map, contouring 3.5 $\sigma$ above mean value) of one of the two tetrasaccharide groups connected to residue 4 .

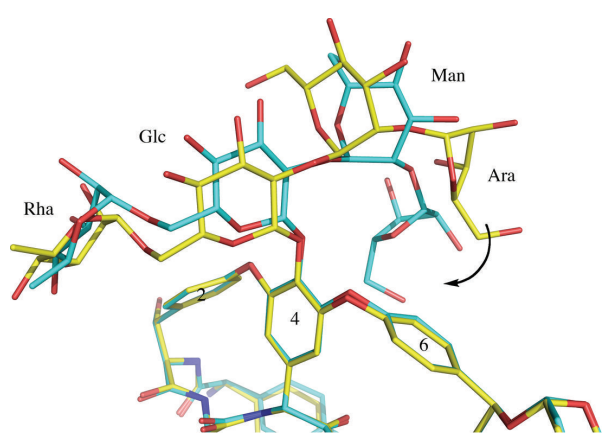

(a)

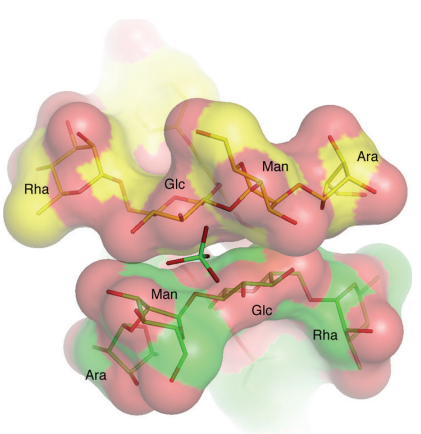

(b)

Figure 3: (a) The tetrasaccharide conformations and orientations in the ligand-free ristocetin-A. Superposition of the two subunits of the homodimer of ligand-free ristocetin-A using $\mathrm{C} \alpha$ of the two aglycon moieties as target by least squares (LSQAB program). The two aglycons are closely superposed but this is not the case for the tetrasaccharide connected to ring \#4. Colors for carbon atoms are in yellow and cyan for the "extended" and "bent" conformations, respectively. (b) The cleft defined by the two tetrasaccharides on top of the dimer (colored in yellow and green). View along the local twofold axis building the homodimer and positioning the two saccharides head-to-tail (anti/anti) represented as Van der Waals surfaces. In this cleft sits the sulfate anion. 


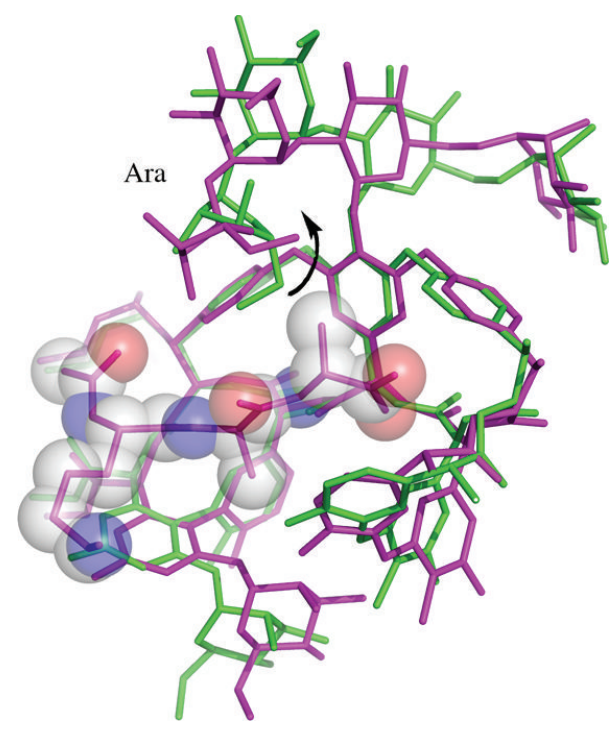

(a)

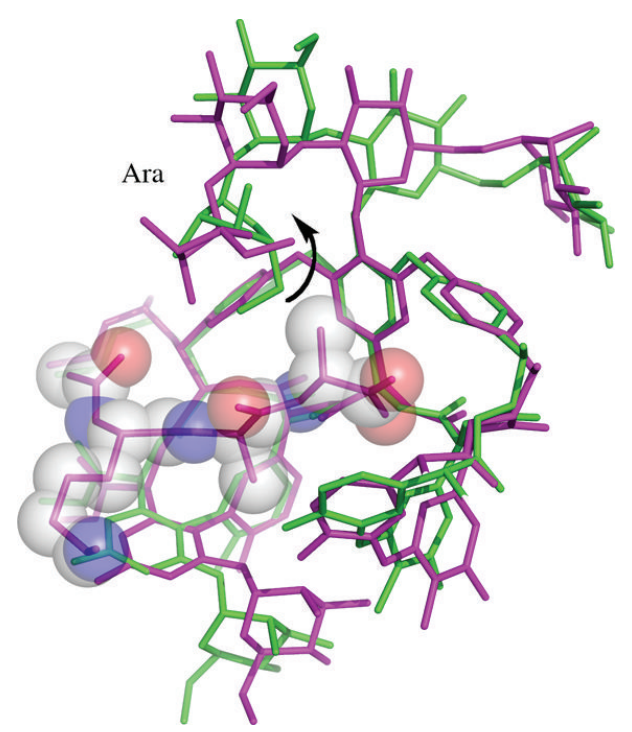

(b)

FIGURE 4: Stereo view of the least squares superposition of the anti conformers of unbound and bound ristocetin-A subunits. In green, the anti(bent) sugar conformation subunit of our monoclinic ligand-free structure; in magenta the anti(bent) monomer of the biliganded structure. The acetyl-tripeptide of the bound form is shown in sphere representation.

Three different structures of ristocetin-A are now available: the free and mono- and biliganded structures. All of them are dimers and hence account for six different molecular views of the ristocetin-A scaffold, all characteristic of the two states of the antibiotic: nonliganded (the two subunits of the present free dimer plus the free subunit connected to its carrier protein at $1.85 \AA$ ) and liganded (the two subunits of the high resolution dimer plus the liganded subunit at $1.85 \AA$ ). A number of features can be inferred when comparing these bound and unbound forms.

Regarding the aglycon, overall shapes are similar with however rms differences in the range of $0.5-0.6 \AA$ relevant to an open/closed adaptation around the ligand. The binding induces the formation of a key $\mathrm{H}$-bond closure between the mannose $\# 13 \mathrm{O}(2)$ and the paraphenolic group of DTyr $1(d=2.82 \AA)$. In the unbound structure, the overall conformation opens and this H-bond no longer exists $(d=$ $5.36 \AA$ ). This open/close conformation was clearly noticed in the monoliganded dimer of ristocetin-A [6].

More dramatic changes are observed in the sugar conformations. To summarize, in the present free structure we have one anti(extended) and one anti(bent) conformations (Figure 3(a)) while the mono- and diliganded structures display conformations syn(bent) and anti(bent). Obviously, the best least squares fits (always using the aglycon $\mathrm{C} \alpha$ 's as target) are obtained with the anti(bent) conformations of the tetrasaccharides ( $\mathrm{rms}-d=0.56 \AA$ for aglycons, but a high degree of freedom can be observed in the sugars with rms$d>2 \AA$, Figure 4 ).

With respect to their lsq superpositions, some important features can be noticed. (1) When comparing the similar anti conformations of the tetrasaccharides, the sugar chain is more bent in the free structure than in the liganded structures; this implies in the bound form a rotation of the
TABLE 2: Mean recalculated isotropic thermal parameters $\langle B\rangle$ in the two tetrasaccharides.

\begin{tabular}{lcc}
\hline $\begin{array}{l}\text { Subunit } \\
\text { Conformation } \\
\text { Mean } B \text { values }\left(\AA^{2}\right)\end{array}$ & $\begin{array}{c}\text { A } \\
\text { Extended }\end{array}$ & $\begin{array}{c}\text { B } \\
\text { Closed }\end{array}$ \\
Rha & & \\
Glc & 6.5 & 6.0 \\
Man & 4.0 & 3.7 \\
Ara & 4.8 & 5.1 \\
\hline
\end{tabular}

arabinose as to let room for the hosted D-peptide ligand (arrow in Figure 4). (2) In the free form, the dynamic behavior of sugars in each tetrasaccharide group suggests a greater mobility for the end-arabinose in the extended configuration than in the bent one as deduced from the thermal factors of sugar atoms (Table 2).

Ristocetin-A was mentioned in solution as a glycopeptide with a unique behavior about interactions with cell wall peptides; two different affinities for the subunits of the dimer, one high and the second much lower, were reported [16]. In all cases, the most efficient binding was associated with the syn orientation of the sugars. In the crystal, these affinities were correlated with allosteric adaptation of the tetrasaccharide that adopts two orientations $180^{\circ}$ apart, the occupied site of high affinity inducing some anticooperativity for the second [6]. In such a hypothesis, this would impose a preliminary dimer/monomer dissociation to allow the rotation of one tetrasaccharide from anti to syn and then redimerization to produce the syn/anti complex observed in the crystal. The structure we describe here represents the free-state of the antibiotic with the two tetrasaccharides in the same anti orientation. We believe the assembly we describe represents 
the ground state of a dimer ready to dissociate and then to interact as individual monomer with the D-Ala-D-Ala sequence, following the pivoting of the tetrasaccharide group from the anti to syn orientation and then inducing a local conformational change of the aglycon around the ligand. The monomer/dimer equilibrium, because of this allosteric modification, would then lead to a dissymmetry in the dimer so as to stay at a low level of energy, with a high affinity site on one side (the syn orientation) and low affinity on the other (the anti orientation).

\section{Conclusion}

The structure of the present ligand-free form of ristocetinA completes the two other syn/anti mono- and biliganded structures already available. Contrary to the liganded structures, it shows a roughly symmetric dimer at the aglycon level, with however a somewhat degree of freedom in the tetrasaccharide moieties. In the liganded structures, the overall conformation of the aglycon expected to be very rigid adapts itself by allosteric changes following the interaction with the target D-Ala-D-Ala sequence of the bacteria cell wall. It becomes evident that the monomer/dimer equilibrium plays a crucial role in leading a bound dissymmetric dimer. The case of the D-Ala-D-Ala sequence included in a long folded peptide chain connected to a large protein [6] would as such represent a good mimic of the bacterial wall complex induced upon ristocetin-A binding.

\section{Competing Interests}

The authors declare that there are no competing interests regarding the publication of this paper.

\section{References}

[1] V. Nahoum, S. Spector, and P. J. Loll, "Structure of ristocetin A in complex with a bacterial cell-wall mimetic," Acta Crystallographica Section D: Biological Crystallography, vol. 65, no. 8, pp. 832-838, 2009.

[2] Y. Nitanai, T. Kikuchi, K. Kakoi, S. Hanamaki, I. Fujisawa, and K. Aoki, "Crystal structures of the complexes between vancomycin and cell-wall precursor analogs," Journal of Molecular Biology, vol. 385, no. 5, pp. 1422-1432, 2009.

[3] C. Lehmann, G. Bunkóczi, L. Vértesy, and G. M. Sheldrick, "Structures of glycopeptide antibiotics with peptides that model bacterial cell-wall precursors," Journal of Molecular Biology, vol. 318, no. 3, pp. 723-732, 2002.

[4] W. G. Prowse, "Conformation of a82846b, a glycopeptide antibiotic, complexed with its cell wall fragment: an asymmetric homodimer determined using nmr spectroscopy," Biochemistry, vol. 34, no. 29, pp. 9632-9644, 1995.

[5] Y.-C. Liu, Y.-S. Li, S.-Y. Lyu et al., "Interception of teicoplanin oxidation intermediates yields new antimicrobial scaffolds," Nature Chemical Biology, vol. 7, no. 5, pp. 304-309, 2011.

[6] N. J. Economou, V. Nahoum, S. D. Weeks et al., "A carrier protein strategy yields the structure of dalbavancin," Journal of the American Chemical Society, vol. 134, no. 10, pp. 4637-4645, 2012.
[7] N. J. Economou, S. D. Weeks, K. C. Grasty et al., "Structure of the complex between teicoplanin and a bacterial cell-wall peptide: use of a carrier-protein approach," Acta Crystallographica Section D, vol. 69, no. 4, pp. 520-533, 2013.

[8] Collaborative Computational Project 4, "The CCP4 suite: programs for protein crystallography," Acta Crystallographica Section D, vol. 50, no. 5, pp. 760-763, 1994.

[9] G. M. Sheldrick, "A short history of SHELX," Acta Crystallographica Section A: Foundations of Crystallography, vol. 64, no. 1, pp. 112-122, 2007.

[10] I. Usón and G. M. Sheldrick, "Advances in direct methods for protein crystallography," Current Opinion in Structural Biology, vol. 9, no. 5, pp. 643-648, 1999.

[11] G. M. Sheldrick, P. G. Jones, O. Kennard, D. H. Williams, and G. A. Smith, "Structure of vancomycin and its complex with acetyl-D-alanyl-D-alanine," Nature, vol. 271, no. 5642, pp. 223225, 1978.

[12] P. T. Beurskens, G. Beurskens, R. de Gelder, S. Garcia-Granda, R. O. Gould, and J. M. M. Smits, The DIRDIF2008 Program System, Crystallography Laboratory, University of Nijmegen, Nijmegen, Netherlands, 2008.

[13] W. L. DeLano, The PyMOL Molecular Graphics System, DeLano Scientific LLC, Palo Alto, Calif, USA, 2002.

[14] V. Nahoum, S. Spector, and P. J. Loll, "Erratum in Structure of ristocetin A in complex with a bacterial cell-wall mimetic," Acta Crystallographica D: Biological Crystallography, vol. 67, part 6, p. 592, 2011.

[15] P. Groves, M. S. Searle, J. P. Mackay, and D. H. Williams, “The structure of an asymmetric dimer relevant to the mode of action of the glycopeptide antibiotics," Structure, vol. 2, no. 8, pp. 747754, 1994.

[16] Y. R. Cho, A. J. Maguire, A. C. Try, M. S. Westwell, P. Groves, and D. H. Williams, "Cooperativity and anti-cooperativity between ligand binding and the dimerization of ristocetin A: asymmetry of a homodimer complex and implications for signal transduction," Chemistry and Biology, vol. 3, no. 3, pp. 207-215, 1996. 

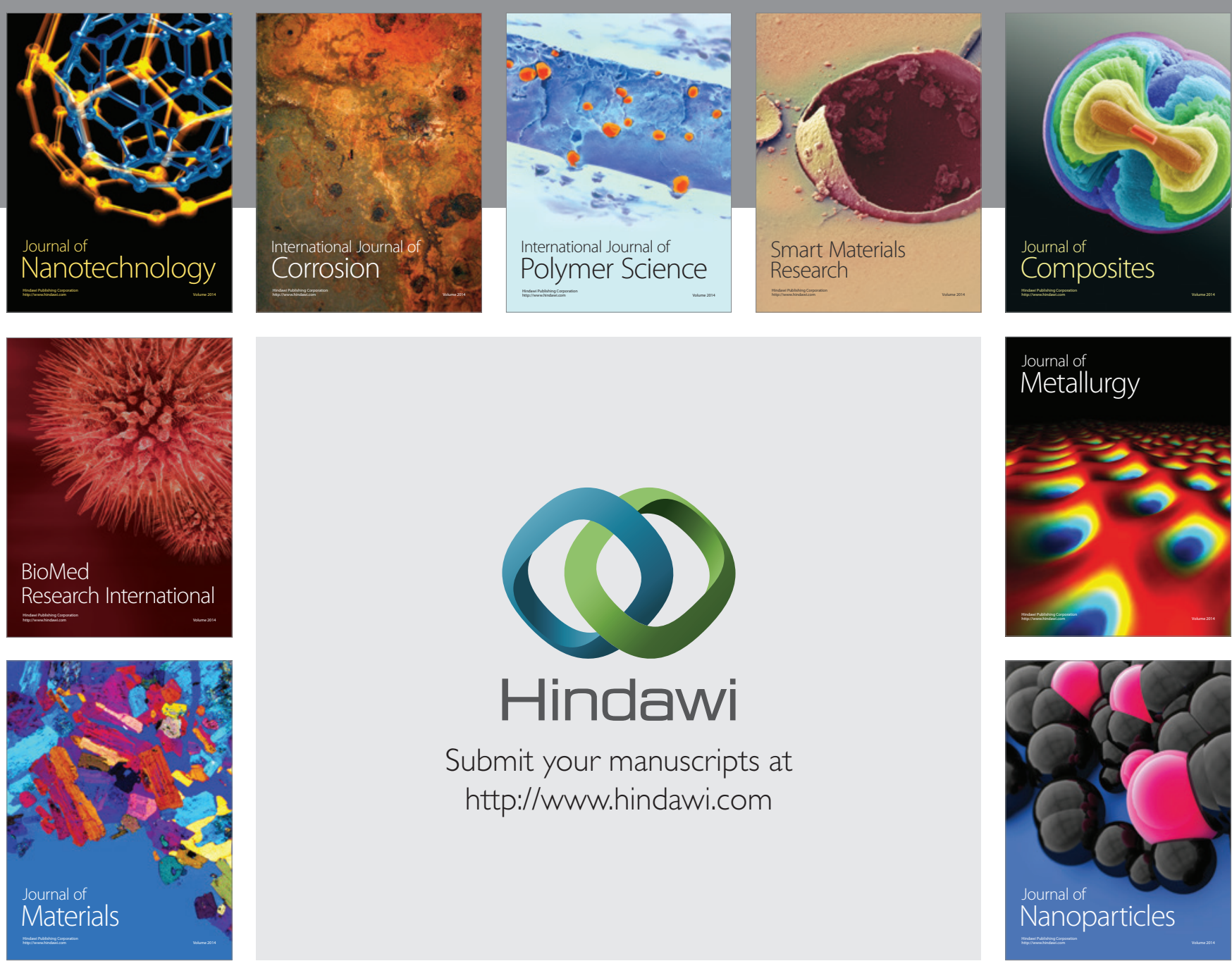

\section{Hindawi}

Submit your manuscripts at

http://www.hindawi.com

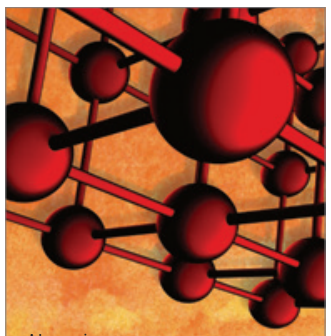

Materials Science and Engineering
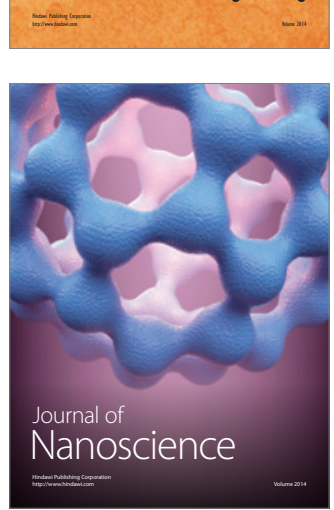
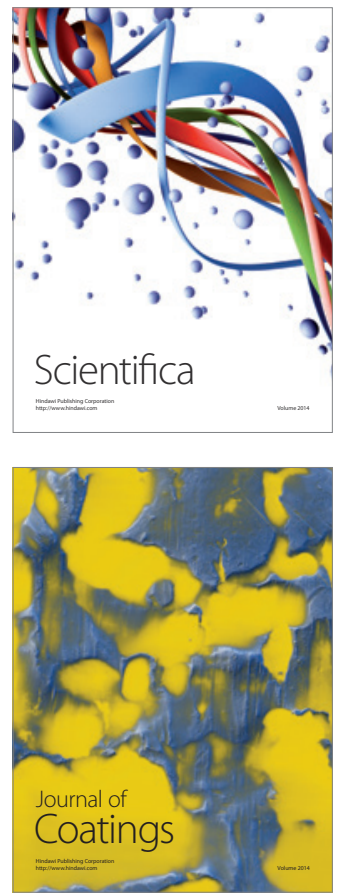
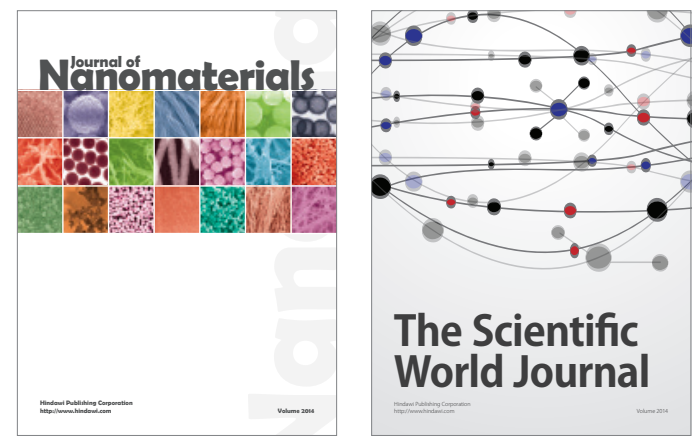

The Scientific World Journal
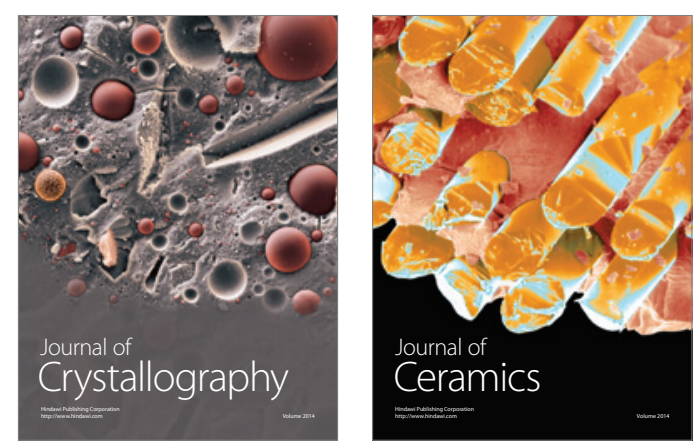
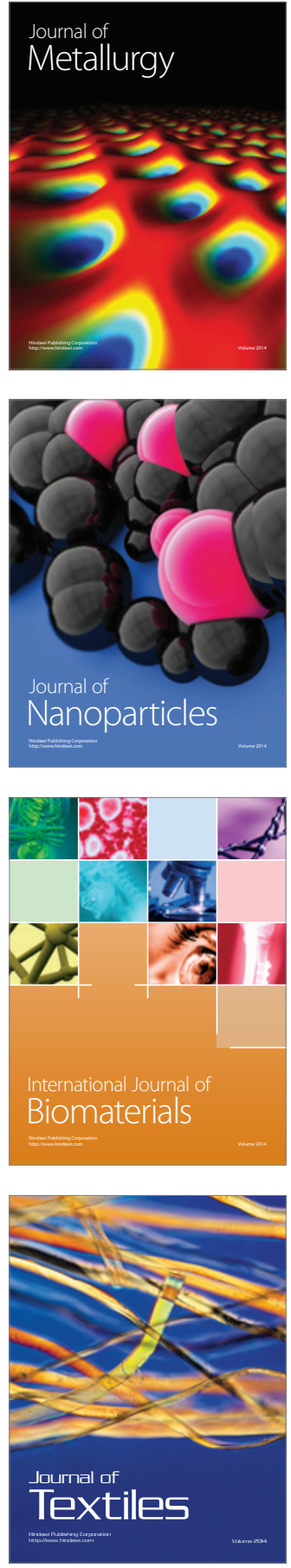